\title{
Long telomeres and well preserved proliferative vigor in cells from centenarians: A contribution to longevity?
}

\author{
C. Franceschi ${ }^{1,2}$, C. Mondello ${ }^{3}$, M. Bonafè ${ }^{1}$, S. Valensin ${ }^{1}$, P. Sansoni ${ }^{4}$, and S. Sorbi ${ }^{5}$ \\ ${ }^{1}$ Department of Experimental Pathology, University of Bologna, Bologna, 2Department of Gerontology, \\ INRCA, Ancona, ${ }^{3}$ Institute of Biochemical and Evolutionary Genetics, CNR, Pavia, ${ }^{4}$ Institute of Internal \\ Medicine, University of Parma, Parma, ${ }^{5}$ Department of Neurological and Psychiatric Sciences, University of \\ Firenze, Firenze, Italy
}

Some years ago, we started investigating healthy people older than 100 years of age as the most reliable model for the study of physiological aging in humans. This research allowed us to discover several paradoxes and to question old tenets in gerontology.

The first, most important result which emerged from these studies is that physiological aging appears to be a process characterized by a profound reshaping, in which several functions and parameters decrease while others increase or remain unchanged $(1,2)$. Accordingly, we proposed a remodeling theory of aging which suggests that the shortage of resources resulting from the deteriorative changes occurring with age is counteracted by a network of anti-aging mechanisms, and optimized by a continuous, complex, adaptive remodeling $(3,4)$. Thus, adaptation and optimization are the key words to understand aging, which appears to be a much more complex phenomenon than previously thought.

Our second major finding can be regarded as a paradox. Indeed, the biochemical parameters and genetic markers, which are currently considered as relevant risk factors for major age-related diseases such as cardiovascular disease, cancer and dementia, among others - were present in healthy centenarians, and their values or frequency were indistinguishable from those of young people (6-8). It is remarkable that healthy centenarians never suffered, at least in a clinically overt way, from the above mentioned diseases, during their long life.

Another paradox emerging from our investigation regards the proliferative capability of cells from healthy centenarians. Our studies focused on two cells types, i.e., peripheral blood lymphocytes (PBL), and fibrob- lasts from skin biopsies (9-12). As far as PBL are concerned, it is well known that an inverse correlation exists between their proliferative capability and the age of the donor. In this type of studies, PBL are usually stimulated by potent mitogenic lectins such as phytohemoagglutinin (PHA); when PBL from centenarians were studied and stimulated by PHA, they followed the age trend, and the number of cells entering the cell cycle was much reduced compared to that of young and middle-aged controls. However, when PBL were stimulated with simpler and more defined stimuli, such anti-CD3 or phorbol myristate acetate, their proliferative capability was unexpectedly well preserved and indistinguishable from that of young people. This result suggests that in centenarians the signal transduction pathway involved by such stimuli is basically intact. Again, the scenario is much more complex than that predicted by a simplified assumption of global failure of proliferative capability with age. Another lesson from these studies is that complex mitogenic stimuli, such as PHA, cannot be considered as the gold standard for assessing possible alterations in the proliferative capability of lymphocytes from elderly people. A bactery of mitogenic stimuli is recommended in order to better assess the effectiveness of the different signal transduction pathways.

The fibroblast is another cell type that has been widely employed to assess the relationship between aging and cell proliferation. The most widely used model has been the culture of normal human fibroblasts, following the seminal observation of Hayflick and Moorehead (13) and Hayflick (14) that these cells display in vitro a limited replicative life span. This phenomenon, described as in vitro senescence, appears

Correspondence: C. Franceschi, M.D., Dipartimento di Ricerche Gerontologiche, INRCA, Via Birarelli 8, 60121 Ancona, Italy. 
to be a general one, and has been observed in a variety of cells types, including lymphocytes. However, the biological relevance of in vitro senescence to the understanding of the mechanisms of organismic aging is still controversial (15). A strong argument in favor of a profound relationship between in vitro senescence and in vivo aging was provided by data suggesting the existence of an inverse correlation between cell proliferative capability and donor age in several cell types, including fibroblasts (see references in 9, 10 and 15). Based on these assumptions, a highly reduced proliferative capability of fibroblasts derived from very old people, such as centenarians, could be predicted. Unexpectedly, we found that the in vitro replicative life span of fibroblast cultures obtained from centenarians was indistinguishable from that of young subjects (10). Moreover, no differences between fibroblast cultures from young and centenarians emerged when the saturation densities of confluent cultures were also considered. On the whole, our data showed that centenarian fibroblasts had growth fractions (number of proliferating cells / total number of cells) and mean population doubling times comparable to cells from young donors, when cultured in medium containing $10 \%$ fetal bovine serum (FBS). When serum-starved fibroblast cultures from centenarians and young donors were stimulated by $20 \%$ FBS, the capacity to complete the different phases of the cell-cycle was equivalent, and the kinetics of the proliferative process were similar. The proliferative responsiveness to different growth factors (b-FGF, EGF, IGF-1, IGF-2) in serum-deprived quiescent cell cultures from centenarians and controls was also very similar, as was the decrease in proliferation during in vitro aging. A possible bias of these studies is a selective capability of "younger" cells to migrate from the explant. In our experience, fibroblast migration from the explants of centenarians was not slower than that of cells from younger donors. Confluent monolayers were reached after about 4 weeks of cultures in all cell strains (10).

The integrity of the cell proliferative machinery in fibroblasts from very old donors is further supported by our investigation of c-fos and c-jun expression, and AP-1 activation in skin fibroblasts from centenarians (9). The cellular proliferative response to growth factors is characterized by the expression of so-called "early genes", among which c-fos and c-jun are the best studied. Several authors reported a decline in seruminduced c-fos expression during in vitro aging of fibroblasts correlated with a progressive loss of proliferative capability. This phenomenon finally results in a growth-arrested state, characterized by a lack of c-fos inducibility after serum stimulation (see ref- erences in 9). Fos and Jun proteins can homo- or hetero-dimerize to form AP-1 (activator protein factor 1) Transcription Factor (TF). AP-1 DNA-binding activity was reported to be deficient in serum-stimulated senescent fibroblasts (see references in 9). Moreover, a reduced basal level of DNA-binding activity has been reported for another inducible TF, such as CREBP (cAMP response element-binding protein), when in vitro aged fibroblasts were compared to early-passage counterparts (see references in 9). We provided evidence that the well-preserved proliferative response of fibroblasts from centenarians is likely due to the fact that some pivotal regulators of cell proliferation, such as c-fos, c-jun and AP-1, are still fully inducible and follow the same induction kinetics of fibroblasts from young donors, despite a long process of in vivo aging (9). Moreover, CREBP DNA-binding activity was constitutively detectable, and quite similar at different time intervals in fibroblasts from young donors and centenarians (9). Our data on centenarians fully agree with those recently observed by Cristofalo et al. in 124 skin fibroblast cultures from healthy donors, ranging in age from the fetal period to 94 years (16). These authors found a high variability in the replicative potential of different cell lines (as we also did), but no clear correlation between donor age and fibroblast replicative life span.

Recently, a molecular clock that keeps track of cell division, i.e., telomere shortening, as a consequence of human aging, has been identified in vivo in normal somatic tissues as well as in in vitro cultured human fibroblasts. Several data indicate that the attrition of telomeres is critical for fibroblast senescence, and that telomere shortening occurs during in vitro propagation of somatic cells $(17,18$; see also references in 12). Moreover, recent data are also consistent with a model in which the maintenance of telomere length may function to support the capacity of lymphocytes for cell proliferation and extensive clonal expansion (19). Telomeres are essential for chromosome stability by protecting chromosome ends from the attack of nucleolytic enzymes, and preventing chromosomes from fusing together. Indeed, the telomere hypothesis of cellular senescence proposes that telomere shortening acts to count cell divisions, and signals the entry to senescence when telomeres fall below a critical length. The causal role of telomere shortening in cellular senescence has been demonstrated by Bodnar et al., who showed that telomerase reactivation induces telomere elongation, and prolongs the life span in normal human cell cultures (20). A critical question regards the relevance of this remarkable in vitro observation to in vivo aging at the organismic level. Assuming a pervasive corre- 
lation between in vivo aging and telomere length, we should expect a consistent shortening of telomeres in all cells derived from centenarians. However, several investigations we have recently performed suggest that this is not the case (12).

Our main results can be summarized as follows: i) unexpectedly, no correlation between telomere length and donor age in early passage skin fibroblasts was found; ii) as expected, telomere shortening during in vitro aging of centenarian fibroblasts occurred; iii) no telomeric fusion was detected in centenarian fibroblasts at early passages, but this phenomenon was evident in cells approaching in vitro senescence; iv) as expected, an inverse correlation between telomere length and donor age in PBL was found. In particular, average telomere shortening was about 30 $\mathrm{bp} /$ year, similar to that reported in the literature. However, this rate apparently was not linear during the entire human life span, i.e., $42.6 \mathrm{bp} /$ year during adulthood (26-29 years of age) and early aging (50-72 years of age), and $15.6 \mathrm{bp} /$ year in older people including nonagenarians and centenarians; v) the direct comparison of telomere length in fibroblasts and PBL from the same individuals showed that shorter telomeres were present in PBL compared to fibroblasts; vi) the analysis of three genes known to be up-regulated during in vitro senescence showed that, apart from fibronectin, no clear correlation exists between expression of ApoJ and p21 in fibroblasts, and the age of the donors, including centenarians.

The main conclusions which can be drawn from these data are the following:

1) the body of old and very old people is a mosaic of cells with different replicative histories and replicative potentials;

2) the inverse correlation between donor age and telomere shortening in bulk lymphocytes in the peripheral blood likely reflects major phenomena of immunosenescence, i.e., the marked decline with age of virgin, naive lymphocytes and the concomitant marked accumulation of memory cells, as well as the shrinkage of the T-cell repertoire $(20,21)$. Memory lymphocytes have undergone several rounds of replication, and thus the presence of short telomeres is expected; and

3) the replicative history of dermal fibroblasts is likely different from that of lymphocytes; indeed, the number of replications of this cell type during in vivo aging and in the skin of centenarians is not known, and has not been properly addressed. Our data suggest that fibroblasts from centenarians show some characteristics of aged cells (altered gene expression) despite having long telomeres, likely due to few replicative rounds.
On the whole, our findings on the replicative capability of cells derived from centenarians can be taken as additional evidence in favor of the hypothesis that in vitro and in vivo aging are neither identical, nor superimposable phenomena. Their possible relationship(s) has (have) to be ascertained very carefully and critically. Moreover, our findings agree with the data we collected on the immunobiology (24) and genetics (25) of centenarians, which showed that a variety of parameters, responses and markers are remarkably preserved and/or present in these individuals. These data cast some doubt on the theories and conceptualizations that still maintain that aging is a catastrophically pervasive phenomenon. Within this scenario, a well-preserved proliferative capability of fibroblasts and lymphocytes might contribute to physiological aging (without major age-related diseases) and longevity.

\section{ACKNOWLEDGEMENTS}

This work and the original data quoted in this paper have been supported by grants to C.F. from the following: EU, BIOMED IV (GENEAGE Project BMH4-CT98-3149; AIRC (Project "Healthy centenarians as a model to study genetic and cellular factors involved in cancer susceptibility"); and the Italian Ministry of Health (IRCCS Project "La prevenzione della morbilità per malattie croniche dell'età avanzata: il modello dei centenari").

\section{REFERENCES}

1. Mariotti S., Franceschi C., Cossarizza A., Pinchera A.: The aging thyroid. Endocr. Rev. 16: 686-715, 1995.

2. Franceschi C., Monti D., Sansoni P., Cossarizza A.: The immunology of exceptional individuals: the lesson of centenarians. Immunol. Today 16: 12-16, 1995.

3. Franceschi C.: Cell proliferation and cell death in the aging process. Aging Clin. Exp Res. 1: 3-13, 1989.

4. Franceschi C., Monti D., Barbieri D., Grassilli E., Troiano L., Salvioli S., Negro P., Capri M., Guido M., Azzi R., Sansoni P., Paganelli R., Fagiolo U., Baggio G., Donazzan S., Mariotti S., D'Addato S., Gaddi A., Ortolani C., Cossarizza A.: Immunosenescence in humans: deterioration or remodelling? Int. Rev. Immunol. 12: 57-74, 1995.

5. Cossarizza A., Ortolani C., Monti D., Franceschi C.: Cytometric analysis of immunosenescence. Cytometry 27: 297313, 1997.

6. Mari D., Mannucci P.M., Duca F., Bertolini S., Franceschi C.: Mutant factor V (Arg506Gln) in healthy centenarians. Lancet 347: 1044, 1996.

7. Mannucci P.M., Mari D., Merati G., Peyvandi F, Tagliabue L., Sacchi E., Taioli E., Sansoni P., Bertolini S., Franceschi C.: Gene polymorphisms predicting high plasma levels of coagulation and fibrinolysis proteins: a study in centenarians. Arterioscler. Thromb. Vasc. Biol. 17: 755-759, 1997.

8. Baggio G., Donazzan S., Monti D., Mari D., Martini S., Gabelli C., Dalla Vestra M., Previato L., Guido M., Pigozzo S., Cortella I., Crepaldi G., Franceschi C.: Lipoprotein(a) and lipoprotein profiles in healthy centenarians: a reappraisal of vascular risk factors. FASEB J. 12: 433-437, 1998. 
9. Grassilli E., Bellesia E., Salomoni P., Croce M.A., Sikora E., Radziszewska E., Tesco G., Vergelli M, Latorraca S., Barbieri D., Fagiolo U., Santacaterina S., Amaducci L., Tiozzo R., Sorbi S., Franceschi C.: C-fos/c-jun expression and AP-1 activation in skin fibroblasts from centenarians. Biochem. Biophys. Res. Commun. 226: 517-523, 1996.

10. Tesco G., Vergelli M., Grassilli E., Salomoni P., Bellesia E., Sikora E., Radziszewska E., Barbieri D., Latorraca S., Fagiolo U., Santacaterina S., Amaducci L., Tiozzo R., Franceschi C., Sorbi S.: Growth properties and growth factor responsiveness in skin fibroblasts from centenarians. Biochem. Biophys. Res. Commun. 244: 912-916, 1998.

11. Sansoni P., Fagnoni F., Vescovini R., Mazzola M., Brianti V., Bologna G., Nigro E., Lavagetto G., Cossarizza A., Monti D., Franceschi C., Passeri M.: T lymphocyte proliferative capability to defined stimuli and costimulatory CD28 pathway is not impaired in healthy centenarians. Mech. Aging Dev. 96: 127136, 1997.

12. Mondello C., Petropoulou C., Monti D., Gonos E.S. Franceschi C., Nuzzo F.: Telomere length in fibroblasts and blood cells from healthy centenarians. Exp. Cell Res. 248: 234-242, 1999.

13. Hayflick L., Moorhead P.: The serial cultivation of human diploid cell strains. Exp. Cell Res. 25: 585-621, 1961.

14. Hayflick L.: The limited in vitro lifetime of human diploid cell strains. Exp. Cell Res. 37: 614-636, 1965.

15. Cristofalo V.J.: Cell culture aging: Insights for cell aging in vivo? Aging. Clin. Exp. Res. 11: 1-3, 1999 (Editorial).

16. Cristofalo V.J, Allen T.G., Pignolo R.J., Martin B.G., Beck J.C.: Relationship between donor age and the replicative lifespan of human cells in culture: A reevaluation. Proc. Natl. Acad. Sci. USA 95: 10614-10619, 1998.

17. Allsopp R.C., Chang E., Kashefi-Aazam M., Rogaev E.I., Piatyszek M.A., Dhay J.W., Harley C.B.:Telomere shortening is associated with cell division in vitro and in vivo. Exp. Cell Res. 220: 194-200, 1995.
18. Sedivy J.M.: Can ends justify the means? Telomeres and the mechanisms of replicative senescence and immortalization in mammalian cells. Proc. Natl. Acad. Sci. USA 95: 90789081, 1998.

19. Weng N., Hatchcock K.S., Hodes R.J.: Regulation of telomere length and telomerase in T and B cells: a mechanism for maintaining replicative potential. Immunity 8: 151-157, 1998.

20. Bodnar A.G., Ouelette M., Frolkis M., Holt S.E., Chiu C.P., Morin G.B., Harley C.B., Shay J.W., Lichtsteiner S., Wright W.E.: Extension of life span by introduction of telomerase into normal cells. Science 279: 349-352, 1998.

21. Cossarizza A., Ortolani C., Paganelli R., Monti D., Barbieri D., Sansoni P., Fagiolo U., Forti E., Londei M., Franceschi C.: Age-related unbalance of virgin (CD45RA+) and memory (CD45R0+) cells between CD4+ and CD8+ T lymphocytes in humans: a study from newborns to centenarians. J. Immunol. Res. 4: 118-126, 1992.

22. Cossarizza A., Ortolani C., Paganelli R., Barbieri D., Monti D, Sansoni P., Fagiolo U., Castellani G., Bersani F., Londei M., Franceschi C.: CD45 isoforms expression on CD4+ and $\mathrm{CD} 8+\mathrm{T}$ cells throughout life, from newborns to healthy centenarians: implications for T cell memory. Mech. Aging Dev. 86: 173-195, 1996.

23. Wack A., Cossarizza A., Heltai S., Barbieri D., D'Addato S., Franceschi C., Dellabona P., Casorati G.: Age-related modifications of the human $\alpha / \beta \mathrm{T}$ cell repertoire as a consequence of clonal expansions. Int. Immunol. 10: 1281-1288, 1998.

24. Franceschi C., Cossarizza A.: The reshaping of the immune system with age. Int. Rev. Immunol. 12: 1-4, 1995.

25. Bonafè M., Olivieri F, Mari D., Baggio D., Mattace R., Sansoni P., De Benedictis G., De Luca M., Bertolini S., Monti D., Franceschi C.: p53 variants predisposing to cancer are present in healthy centenarians. Am. J. Hum. Genet. 64: 292-295, 1999. 